\title{
Fabry-Pérot resonances in graphene microstructures: Influence of a magnetic field
}

\author{
M. Ramezani Masir, ${ }^{1, *}$ P. Vasilopoulos, ${ }^{2, \dagger}$ and F. M. Peeters ${ }^{1, \dagger}$ \\ ${ }^{1}$ Departement Fysica, Universiteit Antwerpen, Groenenborgerlaan 171, B-2020 Antwerpen, Belgium \\ ${ }^{2}$ Department of Physics, Concordia University, 7141 Sherbrooke Ouest, Montréal, Québec, Canada H4B 1R6
}

(Received 1 February 2010; revised manuscript received 6 July 2010; published 10 September 2010)

\begin{abstract}
Fabry-Pérot resonances in the transmission through single and double, graphene-based barriers (of height $V$ ) and wells are investigated and their dependence on an applied perpendicular magnetic field. For rectangular barriers the conductance decreases with increasing magnetic field while the resonances weaken (become more pronounced) with increasing magnetic field for $E_{F}<V\left(E_{F}>V\right)$. The position of the resonances exhibit a linear shift with magnetic field which move to lower (higher) energy for $E_{F}<V\left(E_{F}>V\right)$. Compared to semiellipticor Gaussian-shaped barriers they show a smaller number of resonances in the absence of a magnetic field and an overall lower conductance but the resonant structure is more pronounced. The conductance of asymmetric double barriers show two major regions of resonances while the symmetric ones show one, that of three asymmetric barriers three, and so on.
\end{abstract}

DOI: $10.1103 /$ PhysRevB.82.115417

PACS number(s): 73.23.Ad, 73.23.Hk, 73.43.Jn

\section{INTRODUCTION}

Graphene, a one-atom-thick monolayer of graphite with a honeycomb lattice structure, is a new material which has raised tremendous interest in recent years. Charge carriers in a wide single-layer graphene behave like "relativistic," chiral massless particles with a "light speed" equal to the Fermi velocity and possess a gapless, linear spectrum near the $\mathrm{K}$ and $\mathrm{K}^{\prime}$ points. ${ }^{1,2}$ One major consequence is the perfect transmission through arbitrarily high and wide barriers, referred to as Klein tunneling. ${ }^{3-7}$ This complicates the control of electrons by electric fields in this ideal two-dimensional (2D) layer. Electrons cannot be confined, e.g., in an electrically created quantum dot, the dot has no bound states ${ }^{3}$ but may exhibit sharp resonant states which can be viewed as quasibound states. ${ }^{8}$ Another possibility is to use an inhomogeneous magnetic field. It was shown in numerous papers that an inhomogeneous magnetic field confines the usual electrons ${ }^{9}$ and recently the Dirac electrons as well. ${ }^{10-17}$ Many of graphene's properties are reviewed in two recent works, see Ref. 18.

One important aspect of the electronic transmission through quantum structures is the Fabry-Pérot (FP) resonances which are a consequence of the wave nature of the electron. Recently it was shown that such resonances exist for different semi-infinite structures such as barriers or double barriers (wells). ${ }^{19-22}$ In addition, $n-p, n-p-n$, and $p-n-p$ junctions have been fabricated ${ }^{22-31}$ in which Klein tunneling and Fabry-Pérot resonances were observed. ${ }^{23,24}$ In such devices the height of the potential barriers and the position of the Fermi level could be tuned by gate potentials. In Ref. 24 the metallic gates that induced the potential barriers were about 50-100 $\mathrm{nm}$ separated from the graphene layer and the length of the Fabry-Pérot cavity was about $740 \mathrm{~nm}$. With such devices FP resonances were observed in the resistance oscillations. In such $p$ - $n$ junctions the top gate lays on an insulating layer that can decrease the mobility of graphene. In order to avoid this problem one can fabricate $p-n-p$ graphene structures using suspended air-bridge top gates. $^{25}$
The recent theoretical work of Ref. 22 studied the dependence of Fabry-Pérot resonances on magnetic field in the presence of a parabolic gate potential. ${ }^{25}$ In such a system (for $E>0)$ the electronlike states inside the potential region give rise to the FP resonances. Here we consider a potential barrier which has both electronlike $(E>V)$ and holelike $(E<V)$ states for $E>0$, and compare the effect of a magnetic field on the FP resonances of these two different states. We consider single and multiple rectangular barrier structures and concentrate on resonances in the transmission and conductance and their dependence on magnetic field which, to our knowledge, has not been investigated so far. In Sec. II we study Fabry-Pérot resonances for a single and double barrier as an interferometer. In Sec. III we apply a magnetic field (homogeneous in one direction) in a semi-infinite region, solve the Dirac equation, and obtain the eigenfunctions. Then we present results for the transmission and in Sec. IV for the conductance for zero and nonzero magnetic field. We also investigate the effect of the shape of the potential barriers on the resonances. Our concluding remarks follow in Sec. V.

\section{POTENTIAL BARRIER AS A FABRY-PÉROT INTERFEROMETER}

The low-energy quasiparticles (electrons and holes) in graphene are described by the following Dirac type Hamiltonian:

$$
H=v_{F} \boldsymbol{\sigma} \cdot \mathbf{p}+V,
$$

where $p_{\nu}=-i \hbar\left(\partial / \partial x_{\nu}\right)$ is the momentum operator, $v_{F}$ the Fermi velocity, and $\boldsymbol{\sigma}$ the Pauli matrices. We assume $V=V(x)$ to be one-dimensional potential. Then the equation $H \Psi(x, y)=E \Psi(x, y)$ admits spinor solutions of the form

$$
\Psi(x, y)=\left(\begin{array}{c}
\psi_{\mathrm{I}}(x, y) \\
\psi_{\mathrm{II}}(x, y)
\end{array}\right) .
$$

Due to the translational invariance along the $y$ direction we attempt solutions of the form 

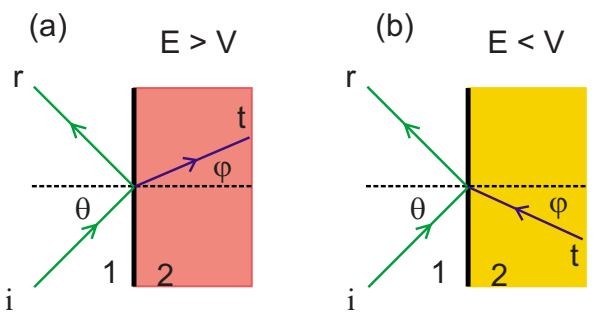

FIG. 1. (Color online) (a) Schematics of the transmission through a potential step for an electron with $E>V$ or positive refractive index. (b) Same as in (a) but for a negative refractive index or $E<V$.

$\Psi(x, y)=\exp \left(i k_{y} y\right)\left[\phi_{1}(x), \phi_{2}(x)\right]^{T}$ with $T$ denoting the transpose of the row vector. Then $\phi_{1}(x)$ and $\phi_{2}(x)$ obey the coupled first-order differential equations,

$$
\begin{aligned}
& -i \hbar v_{F}\left[d / d x+k_{y}\right] \phi_{2}=\varepsilon \phi_{1}, \\
& -i \hbar v_{F}\left[d / d x-k_{y}\right] \phi_{1}=\varepsilon \phi_{2},
\end{aligned}
$$

where $\varepsilon=E-V$. To simplify the notation we introduce the dimensionless variables $t \rightarrow t \ell / v_{F}, \vec{r} \rightarrow \ell \vec{r}, \vec{v} \rightarrow v_{F} \vec{v}, V$ $\rightarrow E_{0} V, E \rightarrow E_{0} E, E_{0}=\hbar v_{F} / \ell$. For a step potential $V(x)$ $=V \Theta(x)$ [see Fig. 1(a)] the solutions of Eqs. (3) are

$$
\begin{gathered}
\Psi_{1}(x, y)=e^{i k_{y} y}\left(\begin{array}{c}
e^{i k_{1} x}+r e^{-i k_{1} x} \\
s_{i}\left[e^{i k_{1} x+i \theta}-r e^{-i k_{1} x-i \theta}\right]
\end{array}\right), \\
\Psi_{2}(x, y)=e^{i k_{y} y}\left(\begin{array}{c}
t e^{ \pm i k_{2} x} \\
\pm s_{i} t e^{ \pm\left(i k_{2} x+i \phi\right)}
\end{array}\right),
\end{gathered}
$$

where $s_{i}=\operatorname{sgn}\left(E-V_{i}\right), \quad k_{i}=\left[\varepsilon_{i}^{2}-k_{y}^{2}\right]^{1 / 2}$ with $i=1,2, \quad \tan \theta$ $=k_{y} / k_{1}$, and $\tan \phi=k_{y} / k_{2}$. After matching these solutions at $x=0$ we obtain the transmission probability $T=t t^{*}$ and consider two different cases: (1) $E<V$, leading to

$$
t_{E<V}=\frac{2 \cos ^{2} \theta}{e^{-i \theta}+e^{-i \phi}}, \quad T_{E<V}=\frac{2 \cos ^{2} \theta}{1+\cos (\theta-\phi)},
$$

and (2) $E>V$ that leads to

$$
t_{E>V}=\frac{2 \cos ^{2} \theta}{e^{-i \theta}+e^{i \phi}}, \quad T_{E>V}=\frac{2 \cos ^{2} \theta}{1+\cos (\theta+\phi)} .
$$

Using electron momentum conservation in the $y$ direction at $x=0$, we obtain Snell's law in the form $\sin \theta=(1-V / E) \sin \phi$. For $E<V$ the step potential acts like a medium with negative refractive index [see Fig. 1(b)] and an incident beam of electrons can be focused to the right of the potential step. ${ }^{4}$ The critical angle is $\sin \theta_{c}=(1-V / E)$. For $E<V / 2$ we find that $\theta_{c}$ is imaginary ( $k_{1}$ is imaginary) and thus the electron is confined to the right of the interface at $x=0$.

Next we consider a potential barrier of width $W$. From an optical point of view the barrier is like a medium with refractive index $1-V / E$. When we inject a wave at an angle of incidence $\theta$, it splits into transmitted and reflected waves, the transmitted wave inside the barrier is multiply reflected at the two edges, at $x=0$ and $W$, as shown in Fig. 2. In analogy with optical waves, the difference in the optical paths along

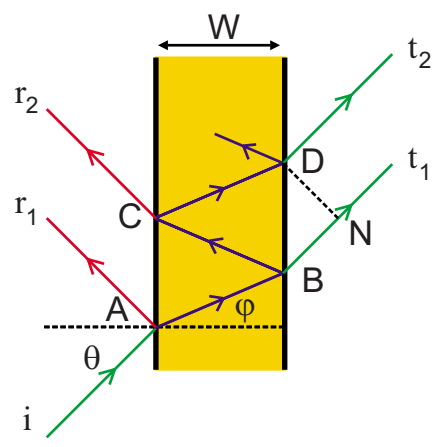

FIG. 2. (Color online) Schematics of multiple reflections inside a barrier.

the barrier, between the transmitted waves $t_{1}$ and $t_{2}$, is

$$
\Delta L=(1-V / E)(B C+C D)-B N,
$$

where $B C=C D=W / \cos \phi$ and $B N=2 W \tan \phi \sin \theta$. Using $\sin \theta=(1-V / E) \sin \phi$, we obtain

$$
\Delta L=2(1-V / E) W \cos \phi .
$$

The total transmission is given by $T=\left|t_{1}\right|^{2}+\left|t_{2}\right|^{2}$ $+2\left|t_{1}\right|\left|t_{2}\right| \cos \delta$ with the corresponding phase difference,

$$
\delta=k_{1} \Delta L=2 k_{1}(1-V / E) W \cos \phi .
$$

The transmission is maximum when $|\delta|=0,2 \pi, 4 \pi, \ldots$, and minimum for $|\delta|=\pi, 3 \pi, \ldots$ To obtain the total transmission we define $r$ and $t$ to be, respectively, the reflection and transmission amplitudes outside the barrier, and $r^{\prime}$ and $t^{\prime}$ the corresponding amplitudes inside the barrier. The different combinations of the transmitted waves through the barrier are

$$
t t^{\prime}, t t^{\prime} r^{\prime 2} e^{i \delta}, \ldots, t t^{\prime} r^{\prime 2(n-1)} e^{i(n-1) \delta}, \ldots,
$$

and the total transmission amplitude

$$
\begin{aligned}
t_{t o t}= & t t^{\prime}\left(1+r^{\prime 2} e^{i \delta}+\cdots+r^{\prime 2(n-1)} e^{i(n-1) \delta}+\cdots\right)=t t^{\prime} /[1 \\
& \left.-r^{\prime 2} e^{i \delta}\right] .
\end{aligned}
$$

The total transmission probability $T_{\text {tot }}=t_{\text {tot }} t_{\text {tot }}^{*}$ is

$$
T_{t o t}=1 /\left[1+F \sin ^{2}(\delta / 2)\right]
$$

with $F=4 R /(1-R)^{2}$ the finesse of the barrier, $R=\left|r^{\prime}\right|^{2}$, and $T=t t^{\prime}$. Using Eqs. (4) and (5) we obtain $r^{\prime}$ and

$$
F=\frac{[1-\cos (\theta-\phi)][1+\cos (\theta+\phi)]}{\cos ^{2} \theta \cos ^{2} \phi} .
$$

Using Eq. (14) in Eq. (13) we find the transmission probability for a single barrier, ${ }^{3,18}$

$$
T=\frac{\cos ^{2} \theta \cos ^{2} \varphi}{[\cos \mu \cos \theta \cos \varphi]^{2}+\sin ^{2} \mu\left(1-s s_{0} \sin \theta \sin \varphi\right)^{2}},
$$

where $\mu=k_{2} W$. Substituting $\mu=n \pi$ in Eq. (15) we obtain the energies $E$ at which the resonances occur (i.e., $T=1$ )

$$
E=V \pm\left[k_{y}^{2}+n^{2} \pi^{2} / W^{2}\right]^{1 / 2} .
$$

We have at least one resonance if 

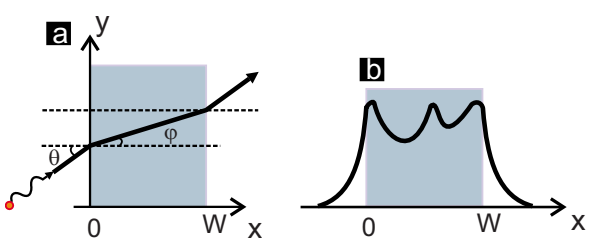

C

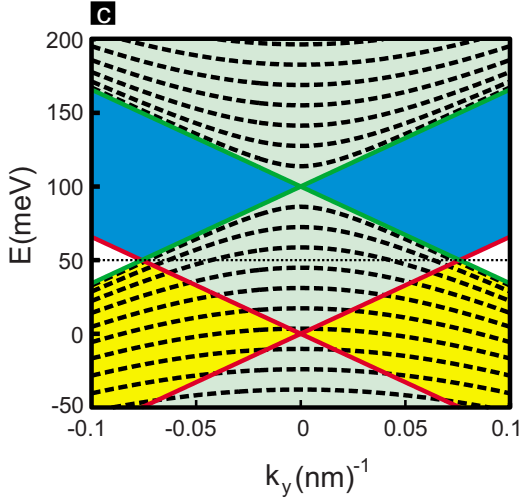

FIG. 3. (Color online) (a) Schematics of the transmission through a barrier of width $W$. (b) The wave function of a localized state in the yellow region of (c). Dispersion relation $E\left(k_{y}\right)$ in the presence of a potential barrier. The dotted curves show the analytical dispersion relation (16) for $W=100 \mathrm{~nm}$ and $V=100 \mathrm{meV}$. The four qualitatively different transmission regions are explained in the text.

$$
W>\pi / V .
$$

As shown in Fig. 3(c) there are four different regions. In the green region $k_{1}$ and $\kappa$ are real and the solutions inside and outside the barrier are traveling waves and due to Klein tunneling we have a high transmission probability where the transmission maxima given by Eq. (16) and shown in Fig. 4(a) by the dashed curves. We can divide this region in two parts: $E>V / 2$ and $E<V / 2$. For $E>V / 2$ all maxima terminate in the free-electron spectrum $E=V \pm k_{y}$ that is shifted by the barrier potential. For $E<V / 2$ though all maxima (dashed curves) cut the free-electron spectrum $E= \pm k_{y}$. The corresponding crossing points can be found by substituting $k_{y}^{2}$ $=E^{2}$ in Eq. (16). We can also obtain the confinement state by using the localized solutions for the electron outside the barrier. The spectrum of the bound states are obtained from the solution of the transcendental equation, ${ }^{32}$

$$
\tan (\kappa W)=-\frac{k_{1} k_{2}}{(V-E) E+k_{y}^{2}} .
$$

Indeed, for $\kappa W=n \pi$, Eq. (18) entails $k_{1} \rightarrow 0, k_{y}^{2}=E^{2}$ and we can rewrite Eq. (16) as

$$
E=\frac{V}{2}-\frac{n^{2} \pi^{2}}{2 W^{2} V} .
$$

In the blue (dark gray) region of Fig. 3(c) $k_{1}$ is real but $k_{2}$ is imaginary. Then we have traveling wave solutions outside the barrier but inside it the solutions are evanescent waves. This is similar to the case of a standard electron with energy
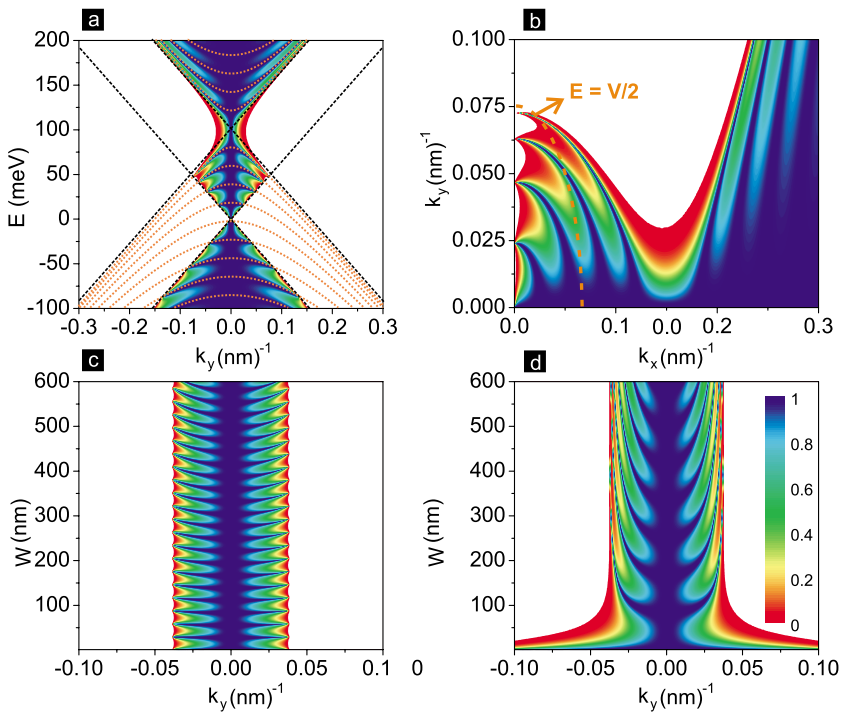

FIG. 4. (Color online) (a) Contour plot of the transmission vs energy $E$ and wave vector $k_{y}$. The dotted curves show the analytical dispersion relation (16) for $W=100 \mathrm{~nm}$ and $V=100 \mathrm{meV}$. (b) Transmission vs $k_{x}$ and $k_{y}$ for $W=100 \mathrm{~nm}$ and $V=100 \mathrm{meV}$. The dashed curve shows the position of $E=V / 2$. [(c) and (d)] Contour plot of the transmission for constant energies $E=25 \mathrm{meV}$ and $E$ $=50 \mathrm{meV}$, respectively, as a function of $W$ and $k_{y}$.

$E<V$ passing through a potential barrier with small transmission probability.

In the yellow region of Fig. 3(c) $k_{2}$ is real but $k_{1}$ is imaginary. This results in traveling wave solutions inside the barrier and evanescent ones outside it, that is, we have states bound to the potential barrier see Fig. 3(b). In fact, we have angular confinement of the waves inside the barriers if the following inequalities are satisfied:

$$
\begin{gathered}
\arctan \left(E_{F} /\left[V^{2}-2 E_{F} V\right]^{1 / 2}\right)<\phi<\pi / 2, \\
-\pi / 2<\phi<-\arctan \left(E_{F} /\left[V^{2}-2 E_{F} V\right]^{1 / 2}\right) .
\end{gathered}
$$

The energies of the bound states coincide with those at which the transmission maxima occur and are given by Eq. (19). Finally, in the white region both $k_{1}$ and $k_{2}$ are imaginary. The corresponding solutions are evanescent waves and the electron cannot tunnel through the barrier.

A few contour plots of the transmission are shown in Figs. 4 and 5, see the caption for details on the parameters. One can clearly see the Fabry-Pérot resonances in all cases. A fundamental difference with the corresponding results for Schrödinger-type electrons is that the Fabry-Pérot resonances now depend on $k_{y}$ as well instead of only the wave vector $k_{x}$. In Fig. 5 we show a contour plot of transmission as a function of momentum and potential height. The potential height can be controlled by an applied top gate. ${ }^{25}$

Next we consider a double barrier and start with a symmetric one, i.e., one in which the barriers have the same height and width and are separated by a distance $W_{s}$. The wave vector $k_{x}$ is the same in the regions between the two barriers, to the left of the first barrier and to the right of the second one. For each barrier we can use the results given 

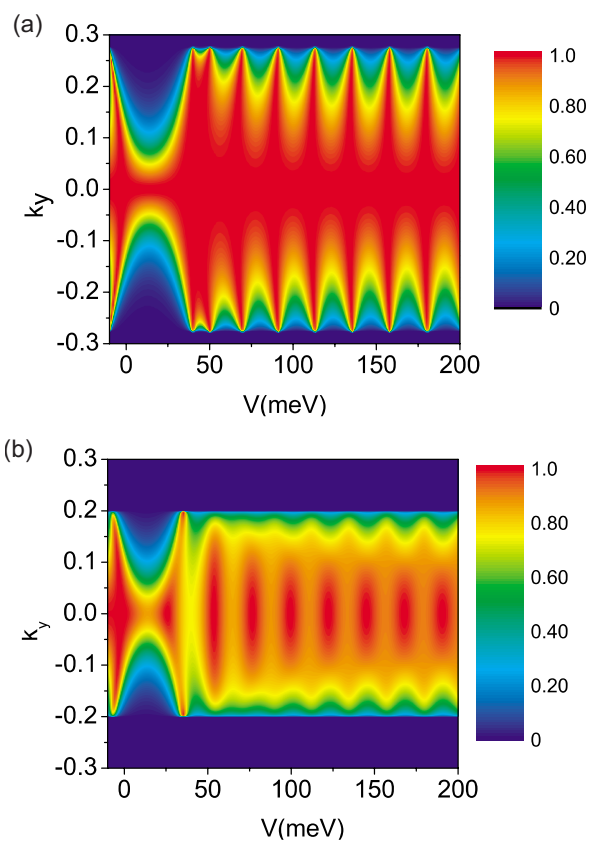

FIG. 5. (Color online) (a) Contour plot of the transmission through a single barrier vs its height $V$ and wave vector $k_{y}$ for $W=100 \mathrm{~nm}$. (b) Same as in (a) but in the presence of a magnetic field $B=100 \mathrm{mT}$.

above. The result for the transmission and reflection coefficients, in matrix form, reads

$$
\left(\begin{array}{l}
1 \\
r
\end{array}\right)=M_{L} M_{W_{s}} M_{R}\left(\begin{array}{l}
t \\
0
\end{array}\right)=M_{D}\left(\begin{array}{l}
t \\
0
\end{array}\right)
$$

here $M_{L}, M_{R}$, and $M_{W_{s}}$ are the transfer matrices to the left of the first barrier, to the right of the second barrier, and between the barriers, respectively. The element $M_{11}$ of the transfer matrix for a single barrier in polar coordinates is

$$
M_{11}=m_{11} e^{i \chi}
$$

where

$$
m_{11}=\left[\cos ^{2}(\kappa W)+\left(\frac{1-s_{0} s \sin \theta \sin \varphi}{\cos \theta \cos \varphi}\right)^{2} \sin ^{2}(\kappa W)\right]^{1 / 2} .
$$

The phase $\chi$ is given by

$$
\chi=-\arctan \left[\left(\frac{1-s_{0} s \sin \theta \sin \varphi}{s \cos \theta \cos \varphi}\right) \tan (\kappa W)\right]+k W,
$$

where $s_{i}=\operatorname{sgn}\left(E-V_{i}\right)$. Evaluating $M_{D 11}$ we obtain

$$
\begin{aligned}
\left|M_{D 11}\right|^{2} & =M_{L 11} M_{R 11} e^{-i k_{x} W}+M_{L 12} M_{R 21} e^{i k_{x} W} \\
& =\left(\left|m_{11}\right|^{2}-\left|M_{21}\right|^{2}\right)^{2}+4\left|m_{11}\right|^{2}\left|M_{21}\right|^{2} \cos \left(k_{x} W_{s}-\chi\right),
\end{aligned}
$$

where the matrix element $M_{21}$ of a single barrier is
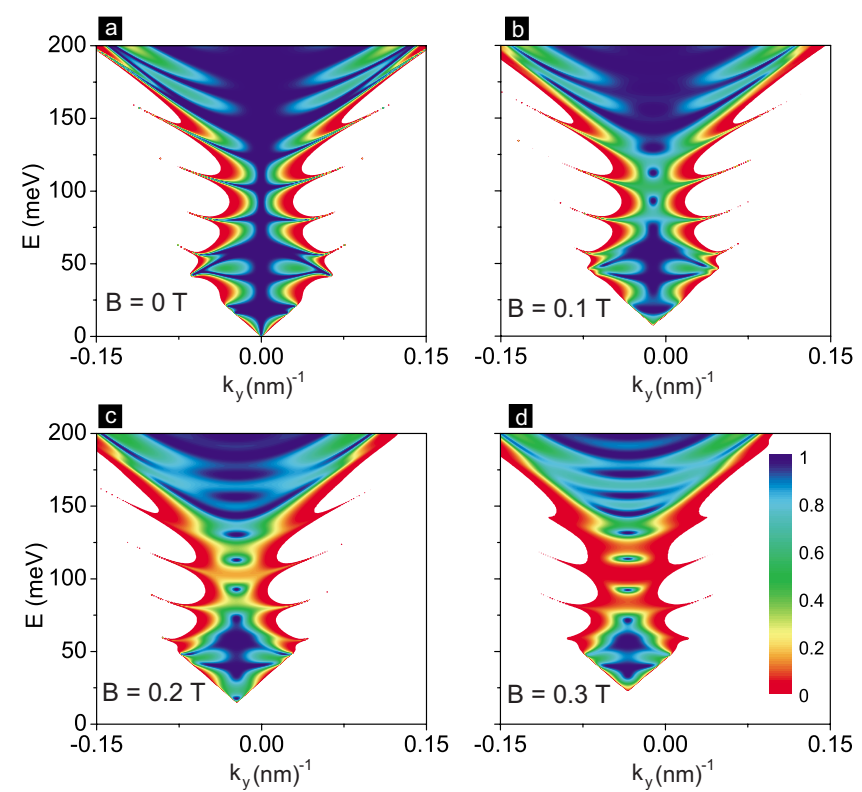

FIG. 6. (Color online) Contour plot of the transmission through a double barrier for different magnetic fields with $W=50 \mathrm{~nm}$, $W_{s}=100 \mathrm{~nm}$, and $V=100 \mathrm{meV}$.

$$
\begin{aligned}
M_{21}= & \frac{1}{2 \cos \theta \cos \varphi}\left[e^{i \theta} \cos (2 \kappa a+\varphi)-\frac{i s_{0}}{s_{1}} \sin (2 \kappa a)\right] \\
& +\left[\frac{i}{s_{1}} \sin (2 \kappa a)-s_{0} e^{i \theta} \cos (2 \kappa a-\varphi)\right]
\end{aligned}
$$

Then the transmission is given by

$$
T_{\text {tot }}(E)=\frac{1}{\left|M_{11}\right|^{2}}=\frac{T_{1}^{2}}{T_{1}^{2}+4 R_{1} \cos ^{2}\left(k_{x} W_{s}-\chi\right)},
$$

where $T_{1}$ and $R_{1}=1-T_{1}$ are the transmission and reflection coefficients of the single barrier [see Eq. (15)], respectively.

The minimum of the transmission occurs for $k_{x} W_{s}-\chi=n \pi$ and is

$$
T_{\text {tot }}^{\min }=\frac{T_{1}^{2}}{T_{1}^{2}+4 R_{1}}=\frac{T_{1}^{2}}{\left(T_{1}-2\right)^{2}} .
$$

This is an increasing function of $T_{1}$ with a maximum value $T_{\text {tot }}^{\min }=1$ when $T_{1}=1$. Notice that here we cannot use the argument that the transmission through the single barrier is small since due to Klein tunneling $T_{1}=1$ for $\kappa W=n \pi$ in which case Eq. (28) gives $T_{\text {tot }}^{\text {min }}=1$. When the cosine in Eq. (28) vanishes the transmission approaches unity which occurs when $T_{\text {tot }}^{\max }=1$,

$$
k_{x} W_{s}-\chi=(2 n+1) \pi / 2, \quad n=0,1, \ldots,
$$

where $W_{s}$ is the distance between the two barriers. Then for a double barrier we have two conditions in order to have maximum transmission, namely,

$$
\kappa W=n \pi, \quad k_{x} W_{s}-\chi=(2 n+1) \pi / 2, \quad n=0,1, \ldots
$$

Results for the transmission are shown in Fig. 6(a) for a double barrier. Notice that the numerical results are rather similar with those of a single barrier depicted in Fig. 4(a) for 

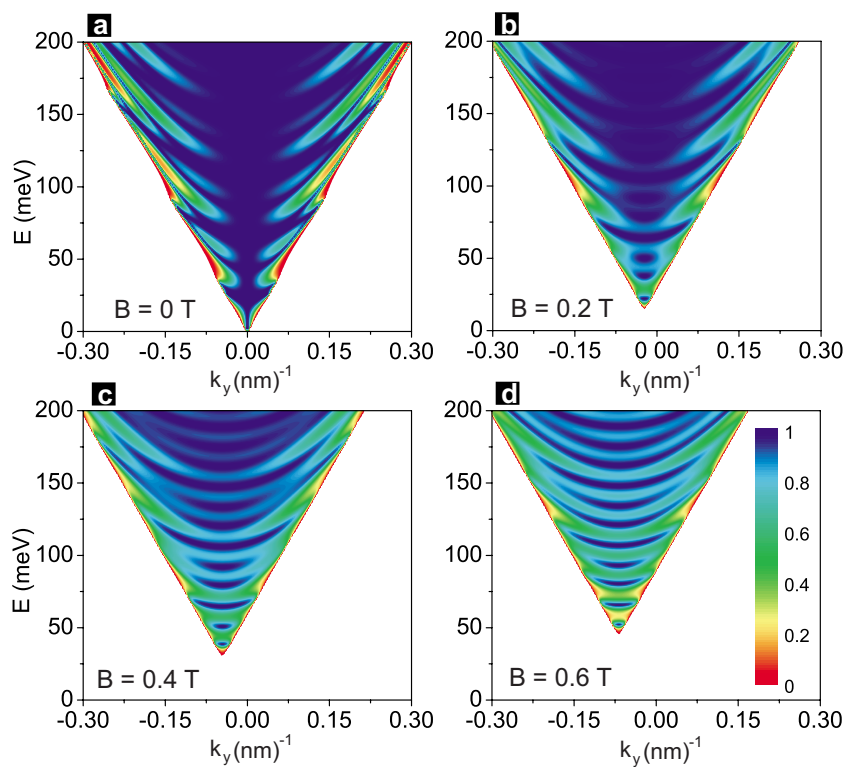

FIG. 7. (Color online) The same as in Fig. 6 but for a double well with $V_{1}=V_{2}=-100 \mathrm{meV}$.

$E>V$. The major difference is that the resonances are more pronounced and are not restricted to the cape region. Notice that for $E<V$ the resonances for a single barrier have a concave $E-k_{y}$ relation while for the resonant structure the behavior is convex (except near $E \sim V / 2$ ).

The above procedure can be repeated for symmetric double wells and the numerical results are shown in Fig. 7(a). As compared to the double barrier structure the resonances are very weak and the transmission is strongly enhanced and different from zero within the cone.

\section{FABRY-PÉROT RESONANCES IN THE PRESENCE OF A MAGNETIC FIELD}

We start with a 2D graphene sheet in the presence of a perpendicular magnetic field $B(x)$ and a constant potential barrier of height $V$ and width $W$. An electron in this system is described by the Hamiltonian

$$
H=v_{F} \boldsymbol{\sigma} \cdot[\mathbf{p}+e \mathbf{A}(x)]+V .
$$

Then $\phi_{1}(x)$ and $\phi_{2}(x)$ obey the coupled first-order differential equations,

$$
\begin{aligned}
& -i \hbar v_{F}\left\{d / d x+\left[k_{y}+e A(x) / \hbar\right]\right\} \phi_{2}=\varepsilon \phi_{1}, \\
& -i \hbar v_{F}\left\{d / d x-\left[k_{y}+e A(x) / \hbar\right]\right\} \phi_{1}=\varepsilon \phi_{2},
\end{aligned}
$$

where $\varepsilon=E-V$. We now consider a homogeneous magnetic field $B_{0}$ and use the Landau gauge $\mathbf{A}(x)=\left(0, B_{0} x, 0\right)$. Again we use the dimensionless variables $B(x) \rightarrow B_{0} B(x), A(x)$ $\rightarrow B_{0} \ell A(x)$ and set $\gamma=e B_{0} \ell^{2} / \hbar=\ell^{2} / \ell_{B}^{2}$, where $\ell_{B}$ is the magnetic length $\left(\ell_{B}=\left[\hbar / e B_{0}\right]^{1 / 2}\right)$ with $\ell=1 \mathrm{~nm}$. Then we can write $A(x)=\gamma x$. Further, by operating on Eq. (31) with $-i\left[d / d x \pm\left(k_{y}+\gamma x\right)\right]$ we can decouple them. With $z=(2 / \gamma)^{1 / 2}\left(k_{y}+\gamma x\right)$ the result is

$$
\left[d^{2} / d z^{2}-z^{2} / 4+p^{\mp}+1 / 2\right] c_{\mp}=0
$$

with $p^{\mp}=\varepsilon^{2} / 2 \gamma \mp 1 / 2-1 / 2, c_{-}=\phi_{1}(z)$, and $c_{+}=\phi_{2}(z)$. The solutions of Eq. (33) are the parabolic cylinder functions which can also be expressed as Weber functions. ${ }^{33}$ The eigenfunctions $c_{ \pm}$are linear combinations of the linearly independent Weber functions $D(p, z)$ and $D(p,-z)$. Explicitly, with $\lambda=i \varepsilon /(2 \gamma)^{1 / 2}$ we have

$$
\begin{gathered}
\phi_{1}(z)=-A \lambda D\left(-\lambda^{2}-1, z\right)+B \lambda D\left(-\lambda^{2}-1,-z\right), \\
\phi_{2}(z)=A D\left(-\lambda^{2}, z\right)+B D\left(-\lambda^{2},-z\right) .
\end{gathered}
$$

In the present paper we are interested in learning how the tunneling resonances are influenced by a magnetic field. We inject an electron beam with momentum $\mathbf{k}=\left(k_{x}, k_{y}\right)$ toward the barrier and investigate the reflected and transmitted beams. If the magnetic field extends over the whole space, such a procedure cannot be used because electrons would be localized on cyclotron orbits. In a real experiment this is overcome by the diffusive motion of the electrons outside the barrier region. Therefore, for technical reasons, we limit the magnetic field to the barrier region, where transport is assumed to be ballistic when we deal with a single barrier, and to the resonant structure region when we deal with a multibarrier structure. First we consider a single barrier, cf. Fig. 3(a). The solutions for the components $\phi_{1}(z)$ and $\phi_{2}(z)$ in the three spatial regions are

$$
\phi_{1}(z)= \begin{cases}e^{i k_{x} x}+r e^{-i k_{x} x} & x<0 \\ \lambda[d D(p-1,-z)-c D(p-1, z)], & 0<x \leq W \\ t e^{i k_{x}^{\prime} x} & x>W,\end{cases}
$$

$$
\phi_{2}(z)= \begin{cases}e^{i k_{x} x+i \phi}-r e^{-i k_{x} x-i \phi} & x<0 \\ c D(p, z)+d D(p,-z) & 0<x \leq W \\ t e^{i k_{x}^{\prime} x+i \varphi} & x>W .\end{cases}
$$

Here $\lambda=i \varepsilon / \sqrt{2 \gamma}, \quad p=\varepsilon^{2} / 2 \gamma, \quad \varepsilon=E-V, \quad k_{x}=\left(E^{2}-k_{y}^{2}\right)^{1 / 2}, \quad k_{x}^{\prime}$ $=\left[E^{2}-\left(k_{y}+\gamma W\right)^{2}\right]^{1 / 2}, \tan \phi=k_{y} / k_{x}$, and $\tan \varphi=\left(k_{y}+\gamma W\right) / k_{x}^{\prime}$. To find the transfer matrix for this system we proceed as follows. For $0<x<W$ the solution can be written as a combination of Weber functions. Matching the wave function at $x=0$ and $x=W$ gives the matrices

$$
\begin{gathered}
N_{1}=\left(\begin{array}{cc}
-\lambda D_{p-1}\left(z_{0}\right) & \lambda D_{p-1}\left(-z_{0}\right) \\
D_{p}\left(z_{0}\right) & D_{p}\left(-z_{0}\right)
\end{array}\right), \\
N_{2}=\left(\begin{array}{cc}
-\lambda D_{p-1}\left(z_{W}\right) & \lambda D_{p-1}\left(-z_{W}\right) \\
D_{p}\left(z_{W}\right) & D_{p}\left(-z_{W}\right)
\end{array}\right) .
\end{gathered}
$$

The transfer matrix $M$ and the transmission $T=1 /\left|M_{11}\right|^{2}$ is determined by its element $M_{11}$ given by

$$
M_{11}=\frac{\left[N_{11}+N_{12} e^{i \theta}\right] e^{2 i k a-i \theta}+\left[N_{21}+N_{22} e^{i \theta}\right] e^{2 i k a}}{2 \cos \theta}
$$

with the matrix $N$ given by $N=N_{1} N_{2}^{-1}$. In a similar manner we obtain the results for a single well. 

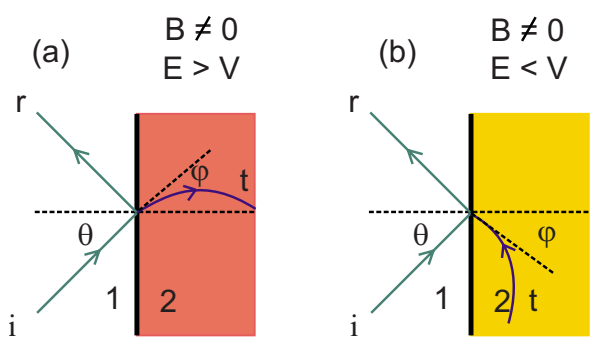

FIG. 8. (Color online) Potential step in the presence of a magnetic field.

In the presence of a magnetic field the transmitted waves inside the barrier will turn and the angles for the transmitted and reflected waves inside the barrier are different (see Fig. 8). The relations between the angle of the transmitted wave inside the barrier $\varphi_{1}$ and the angle of the traveling wave $\varphi_{2}(x)$ inside the barrier are

$$
\int_{\varphi_{1}}^{\varphi(x)} d \varphi \cos \varphi=\int_{0}^{x} d x \frac{\gamma}{E-V}
$$

and thus,

$$
\sin \varphi_{2}(x)=\sin \varphi_{1}+\frac{\gamma x}{E-V},
$$

from which we obtain a relation between the angle of incidence $\theta_{1}$ and the angle of exit $\varphi_{2}(W)$,

$$
\sin \varphi_{2}(W)=\frac{E \sin \theta_{1}+\gamma W}{E-V} .
$$

The critical angle $\theta_{c}$ can be found by setting $\varphi_{2}(W)=\pi / 2$; it is

$$
\sin \theta_{c}=\left(1-\frac{V+\gamma W}{E}\right)
$$

and due to the inequality $-1<\sin \theta_{c}<1$ we find

$$
-\frac{V}{W}<\gamma<\frac{2 E-V}{W}
$$

In the presence of a weak magnetic field the corresponding optical refractive index changes with the position $x$ in the manner $n(x)=1-V_{e f f}(x) / E$. The function $V_{\text {eff }}(x)$ plays the role of an effective potential. Let us consider $V_{\text {eff }}(x)=V+g(x)$, where $V$ is the initial potential at zero magnetic field and $g$ is a perturbation term due to the magnetic field. Using Eq. (41) we can evaluate $g$ as

$$
g(x)=\frac{\gamma(E-V) x}{(E-V) \sin \varphi_{1}+\gamma x} .
$$

For weak magnetic fields $\gamma$ is small and the effective refractive index can be written as

$$
n(x) \approx 1-\frac{V}{E}-\frac{\gamma x}{E \sin \varphi_{1}} .
$$

The classical trajectory between 0 and $W$ can be obtained from

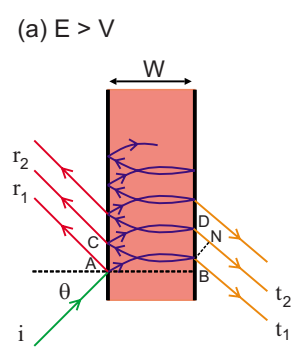

(b) $E<V$

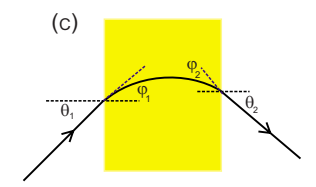

FIG. 9. (Color online) Electron trajectories through a potential barrier in the presence of a magnetic field.

$$
\frac{d^{2} y}{d x^{2}}=\frac{1}{2 \alpha^{2}} \frac{\partial n^{2}}{\partial x}
$$

with $\alpha=(1-V / E) \cos \varphi_{1}$. If we neglect terms of order $\gamma^{2}$ the solution of Eq. (47) is

$$
y=\frac{-\gamma}{2(E-V) \sin \varphi_{1} \cos ^{2} \varphi_{1}} x^{2}+x \tan \varphi_{1} .
$$

Using the same procedure as in Sec. II we obtain the classical optical path,

$$
\Delta L \approx 2\left(1-\frac{V}{E}\right) W \cos \phi-\frac{\gamma W^{2}}{E}\left[\frac{1+2 \sin ^{2} \varphi_{1}}{\sin \varphi_{1} \cos \varphi_{2}}\right],
$$

where the first term is the optical path at zero magnetic field [see Eq. (9)]. As shown in Fig. 9(a) for $E>V$ the optical path decreases with increasing magnetic field while in Fig. 9(b) for $E<V$ it increases. For $E<V, \varphi_{2}$ increases very fast with magnetic field and the probability for the electron to pass through the barrier diminishes quickly. As shown later in Fig. 12 for $E<V$, the resonances weaken with increasing magnetic field and finally disappear.

For double barriers or wells we follow the same procedure. Because the analytical results become too unwieldy, in what follows we present only the numerical results. A contour plot of the transmission through a double barrier, for different magnetic fields, is shown in Figs. 6(a)-6(d) and through a double well in Figs. 7(a)-7(d). We see clearly that increasing the magnetic field leads to a shift $\Delta k_{y}=-\gamma\left(2 W+W_{s}\right) / 2$ (and correspondingly to an induced energy gap $\left.\Delta E / E_{0}=2\left|\Delta k_{y}\right|\right)$ of the transmission cone, a reduction in the number of resonances, and a shrinking of the region of perfect transmission.

\section{CONDUCTANCE}

Selecting the wave vector components $k_{x}$ and $k_{y}$ is very difficult, although, in principle, possible experimentally using quantum point contacts. However, experimentally one usually measures the average transmission. Typically one measures the current $J$ which is proportional to a weighted integral of the transmission $T\left(k_{x}, k_{y}\right)$. For the linear spectrum $E=\hbar v_{F} k$ the conductance $G$ is given by 


$$
G=G_{0} \int T(E, \theta) f(1-f) E d E \cos \theta d \theta,
$$

where $f \equiv f\left(E-E_{F}\right)$ is the Fermi-Dirac distribution function and $E_{F}$ the Fermi level. Further, $G_{0}=2 e^{2} L / v_{F} h^{2}$ and $L$ is the width of the entire structure along the $y$ axis $(L \gg W)$. With the explicit form of $f$ the conductance becomes

$$
\begin{aligned}
G\left(E_{F}\right)= & G_{0} \int_{-\infty}^{+\infty} E d E \int_{-\pi / 2}^{\pi / 2} T(E, \theta) \\
& \times(\beta / 4) \cosh ^{-2}\left[\beta\left(E-E_{F}\right) / 2\right] \cos \theta d \theta
\end{aligned}
$$

with $\beta=1 / k_{B} T$ and $k_{B}$ the Boltzmann constant. Here we will restrict ourselves to zero temperature. Then the conductance takes the simpler form

$$
G=G_{0} \int_{-\pi / 2}^{\pi / 2} T\left(E_{F}, \theta\right) E_{F} \cos \theta d \theta
$$

Here we consider only the conductance through the tunneling barrier(s) and neglect the contribution from the region before and after the resonant structure. Therefore, we only need the transmission $T(E, \theta)$ through the resonant structure where it is allowed to restrict the magnetic field to the region of the resonant barrier structure.

First we consider the conductance for transport through a single barrier. In order to show more clearly the resonances we plot in Fig. 5 the second energy derivative of the conductance as a function of the Fermi energy $E_{F}$ and the width of a single barrier for $E_{F}<V$ in (a) and $E_{F}>V$ in (b). In both cases the magnetic field is zero. The positions of the resonances are approximately given by the dashed curves,

$$
E_{n}=V \pm n \pi / W,
$$

the $+(-) \operatorname{sign}$ is for $E_{F}>V\left(E_{F}<V\right)$. For $E_{F}<V / 2$, that is, to the left of the vertical dashed line in Fig. 10(a), we notice two types of peaks that have a different energy dependence. One is given by Eq. (53) and the other by Eq. (19). The latter increases much faster upon increasing the energy and asymptotically reaches the line $E=V / 2$ for $W \rightarrow \infty$ as we show separately in Fig. 10(c). The latter resonances occur only for $E<V / 2$ and this implies that the transmission maxima are connected to the bound states in the barrier region as given by Eq. (19). With reference to Fig. 4(b) we see that all these maxima occur for $E<V / 2$.

From the Bohr-Sommerfeld quantization condition we have 22,34

$$
\int_{x_{-}}^{x_{+}} p_{x}(x) d x=(n+1 / 2-\zeta) \pi \hbar
$$

with

$$
p_{x}(x)=\left[(E-V)^{2}-\left(k_{y}+\gamma x\right)^{2}\right]^{1 / 2},
$$

where $x_{-}$and $x_{+}$are the turning points, $x_{ \pm}=-k_{y} / \gamma \pm \varepsilon / \gamma, \varepsilon$ $=E-V$, and $\zeta$ the Berry phase contribution which is $1 / 2$ for Dirac fermions. ${ }^{35}$ The integral over $x$ can be carried out. The result is
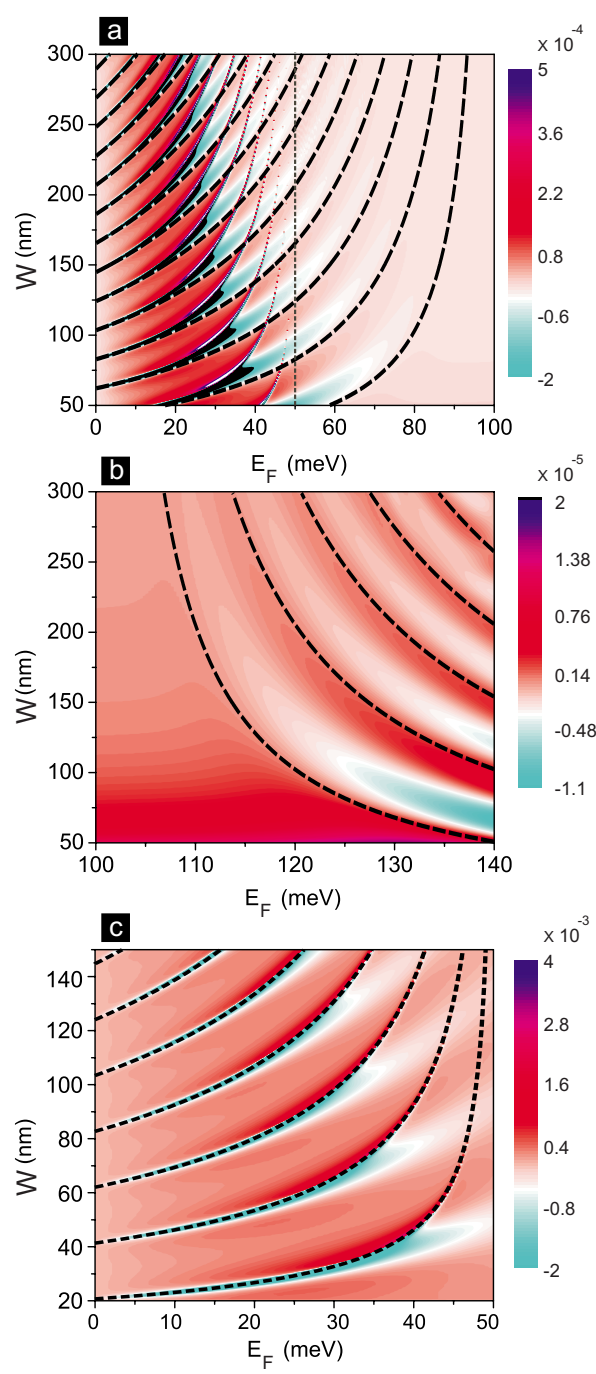

FIG. 10. (Color online) Contour plot of the second derivative of the conductance with respect to the energy for a single barrier vs energy $E$ and barrier width $W$, for $E_{F}<V$ in (a) and $E_{F}>V$ in (b), with $V=100 \mathrm{meV}$. The dashed curves show the position of the peaks resulting from Eq. (53). (c) The additional peaks of (a) for $E_{F} \leq V / 2$.

$$
I=\int_{0}^{W} p_{x}(x) d x=\frac{\varepsilon^{2}}{2 \gamma}\left[y\left[1-y^{2}\right]^{1 / 2}+\arcsin y\right]_{0}^{W}
$$

with $y=\left(k_{y}+\gamma x\right) / \varepsilon$. The transmission probability is symmetric about $k_{y}=-q_{0}=-\gamma W / 2$ and thus we find

$$
I=(\varepsilon / \gamma)\left[q_{0}\left[1-q_{0}^{2}\right]^{1 / 2}+\arcsin \left(q_{0}\right)\right]
$$

For weak magnetic fields $(\gamma \rightarrow 0)$, the integral in Eq. (57) can be simplified to

$$
I \approx \int_{0}^{W}\left[\kappa^{2}-2 k_{y} \gamma x\right]^{1 / 2} d x
$$

This gives 


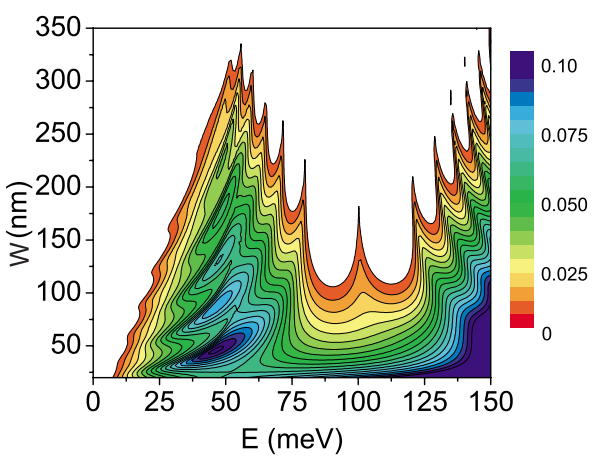

FIG. 11. (Color online) Contour plot of the conductance of a single barrier vs Fermi energy $E_{F}$ and barrier width $W$ for $V=100 \mathrm{meV}$ and $B=0.3 \mathrm{~T}$.

$$
\varepsilon_{n} \approx \pm\left\{\frac{n \pi}{2 W}+\left[\left(\frac{n \pi}{2 W}\right)^{2}+\left(\frac{\gamma W}{2}\right)^{2}\right]^{1 / 2}\right\} .
$$

For $\gamma=0 \mathrm{Eq}$. (59) is exactly the same as Eq. (16) with $k_{y}=0$. The difference between the spectrum with and without magnetic field is

$$
\Delta \varepsilon_{n}=\varepsilon_{n}^{B \neq 0}-\varepsilon_{n}^{B=0} .
$$

Now we consider two different cases:

(1) For $E<V$ we have

$$
\Delta \varepsilon_{n}=n \pi / 2 W-\left[(n \pi / 2 W)^{2}+(\gamma L / 2)^{2}\right]^{1 / 2}
$$

while for $\gamma=0$ we easily obtain $\Delta \varepsilon_{n}=0$. Because of $\Delta \varepsilon_{n}<0$ we have $\varepsilon_{n}^{B \neq 0}<\varepsilon_{n}^{B=0}$. This means that the resonance energies in the presence of a magnetic field shift below those for zero magnetic field. For the resonance energies in the range $0<E<V$ we have also another limitation which comes from the energy gap opening through the magnetic field, $E_{\text {gap }}=\gamma W / 2$. The resonance energies in the presence of a magnetic field can be reduced until the energy value becomes equal to $E_{\text {gap }}$ or simply $E_{\text {gap }}=\varepsilon_{n}^{B \neq 0}$. From this relation we can also find the number of resonances $n$ and the width $W$ after which the conductance drops to zero, see Fig. 11 for $0<E<V$.

(2) For $E>V$ we have

$$
\Delta \varepsilon_{n}=-n \pi / 2 W+\left[(n \pi / 2 W)^{2}+(\gamma L / 2)^{2}\right]^{1 / 2} .
$$

This means that the position of the resonances in the presence of magnetic field is shifted to higher energy or $\varepsilon_{n}^{B \neq 0}>\varepsilon_{n}^{B=0}$, see Fig. 11 for $E>V$.

The conductance of a single barrier and that of a single well are shown in Fig. 12 for different magnetic fields $B$, the former to the right of the point $E_{F}=0$ and the latter to its left. It is apparent from Fig. 12 that the conductance decreases with increasing magnetic field. We also see that there is a special point $E_{F} \approx V$ in the former where the conductance is almost insensitive to the value of the field $B$ and the inset makes that clear. This can be understood as follows. For $E_{F} \rightarrow V$ and $k_{y} \rightarrow \epsilon \rightarrow 0$ Eq. (15) shows that the transmission probability near the top of the barrier is

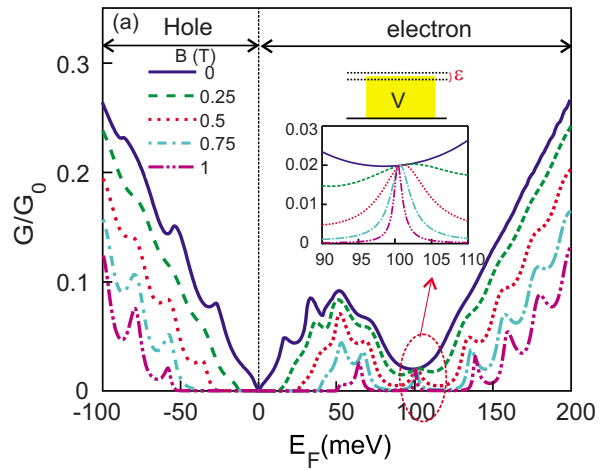

FIG. 12. (Color online) Conductance of a single barrier and a single well for different magnetic fields with $W=100 \mathrm{~nm}$ and $V=100 \mathrm{meV}$. The inset is a blowup of the region near $100 \mathrm{meV}$.

$$
T \approx 1 / \cosh ^{2}\left(k_{y} W\right),
$$

as previously obtained in Refs. 36 and 37 in the absence of a magnetic field. The corresponding conductance is

$$
G=\frac{4 e^{2}}{v_{F} h^{2}} \frac{L}{W} \tanh (V W) .
$$

To see the dependence of the conductance on magnetic field more clearly, we show a contour plot of its logarithm in Fig. 13 for a single barrier. We notice that for $E_{F}>V$ the energy of the different resonances increase with $B$ whereas for $E_{F}<V$ they decrease. This opposite behavior can be understood as follows. In the limiting case $\gamma W \ll p^{2}$, that is, for $B \rightarrow 0$, to a first approximation the resonant energies are

$$
E-E_{n} \sim \pm \gamma / 2 N
$$

with $N=(n+1 / 2) \pi / W$; the $+(-)$ sign is for $E>V(E<V)$ and $E_{n}$ is given by Eq. (53). Accordingly, these energies, as a function of the magnetic field, for $E_{F}>V$ have the opposite behavior compared to those for $E_{F}<V$. A contour plot of the first derivative of the conductance of a $p-n-p$ structure is shown in Fig. 14(a). The resonances in the conductance for several magnetic fields are shown in Fig. 14(b) where we plot the conductance of the $p-n-p$ structure as a function of the potential height for fixed Fermi energy. The results are in good agreement with the experimental ones. ${ }^{23}$

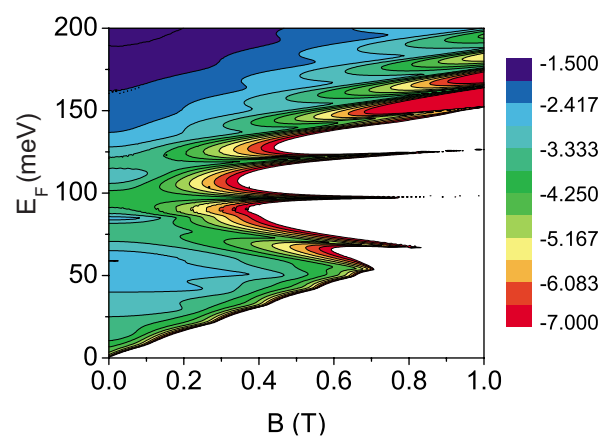

FIG. 13. (Color online) Contour plot of the logarithm of the conductance of a single barrier, in units of $G_{0}$, vs magnetic field $B$ and Fermi energy $E_{F}$ with $W=100 \mathrm{~nm}$. 
(a)
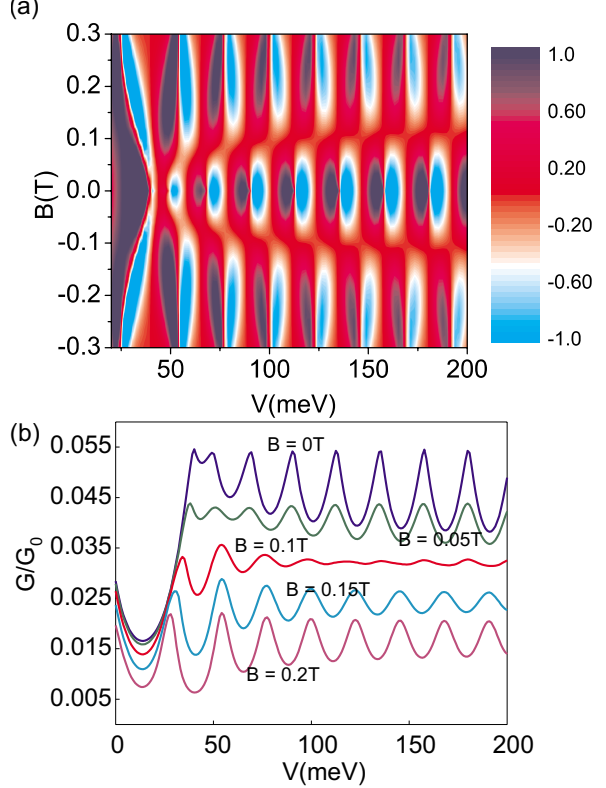

FIG. 14. (Color online) (a) Contour plot of the derivative of the conductance of a $p-n-p$ structure vs potential $V$ and magnetic field for $W=100 \mathrm{~nm}$. (b) Conductance of the structure in (a) vs potential for different magnetic fields $B=0,50,100,150$, and $200 \mathrm{mT}$.

For $E_{F} \approx V$ the conductance for $B=0$ exhibits a local minimum, which is a signature of Klein tunneling. Notice that for $E_{F}<0$ we have holes impinging on a quantum well and the conductance is similar to that for $E_{F}>V$. These resonances for "normal" electrons are known as the Ramsauer effect. We found that they become more pronounced with increasing magnetic field. In the presence of a weak magnetic field, such that $\gamma W \ll V$ holds, the solution inside the barrier can be written as

$$
\Psi_{i n}(x)=\left(\begin{array}{l}
A \exp \left[-\left(k_{y}-\gamma x\right)^{2} / 2 \gamma\right] \\
B \exp \left[-\left(k_{y}+\gamma x\right)^{2} / 2 \gamma\right]
\end{array}\right) .
$$

Matching the solutions at $x=0$ and $x=W$ gives

$$
\left\{\begin{array}{l}
1+r=A \exp \left[-k_{y}^{2} / 2 \gamma\right] \\
e^{i \phi}-r e^{-i \phi}=B \exp \left[-k_{y}^{2} / 2 \gamma\right]
\end{array}\right.
$$

and

$$
\left\{\begin{array}{l}
t e^{i k_{x}^{\prime} W}=A \exp \left[-\left(k_{y}-\gamma W\right)^{2} / 2 \gamma\right] \\
t e^{i k_{x}^{\prime} W+i \varphi}=B \exp \left[-\left(k_{y}+\gamma W\right)^{2} / 2 \gamma\right] .
\end{array}\right.
$$

Then the transmission probability reduces to

$$
T=\frac{4 \cos \varphi \cos \phi e^{k_{y}^{2} / \gamma}}{S_{+}^{2}+S_{-}^{2}+2 S_{+} S_{-} \cos (\phi+\varphi)}
$$

with $S_{ \pm}=\exp \left[\left(k_{y} \pm \gamma W\right)^{2} / 2 \gamma\right]$. For $E \approx V$ and $\gamma W \ll V$ the transmission probability reduces to
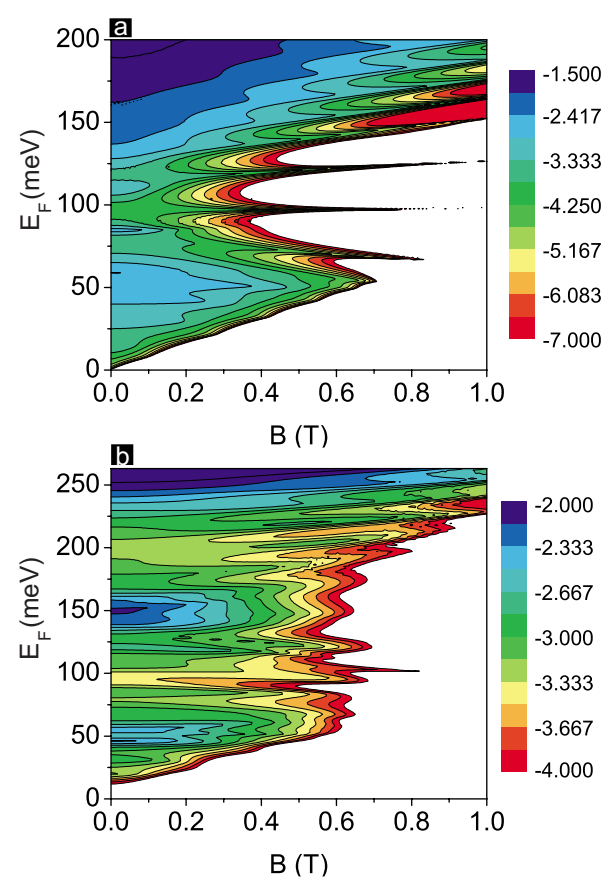

FIG. 15. (Color online) (a) Contour plot of the logarithm of the conductance, in units of $G_{0}$, of a symmetric double barrier vs energy and magnetic field with $W=50 \mathrm{~nm}$, separation $W_{s}=50 \mathrm{~nm}$, $V_{1}=V_{2}=100 \mathrm{meV}$. (b) As in (a) for an asymmetric double barrier with $V_{1}=100 \mathrm{meV}$ and $V_{2}=200 \mathrm{meV}$.

$$
T \approx \frac{e^{-\gamma W^{2}}}{\cosh ^{2}\left(k_{y} W\right)} .
$$

The corresponding conductance is

$$
G=\frac{4 e^{2}}{v_{F} h^{2}} \frac{L}{W} \tanh (V W) e^{-\gamma W^{2}} .
$$

Similar to the single-barrier case, in we show a contour plot of the logarithm of the conductance for a symmetric double barrier in Fig. 15(a) and an asymmetric one in Fig. 15(b). One feature common in both panels is the reduction in the conductance upon increasing $B$ and second an additional region in panel (b), centered around $E_{F}=150 \mathrm{meV}$, in which $G$ is significantly different from zero. We can see these features more clearly in the conductance $G$, shown in Fig. 16, for the symmetric double barrier (top panel) and the asymmetric one (bottom panel) for three values of $B$. For clarity the results for the symmetric case are shifted up by 0.15 . The first feature can be understood as follows. For $B=0$ the wave vector to the left and right of the barrier is the same. This is not the case when $B$ is present because the wave vector component $k_{y}$ is shifted by $\gamma W$ (see Fig. 6). Then the green region in Fig. 3(c) will shrink and so will the transmission and the conductance. The second feature, in the bottom panel, centered around $E_{F}=150 \mathrm{meV}$ in which $G$ is significantly different from zero, can be understood as follows. When the barrier heights $V_{1}$ and $V_{2}$ are equal, there is only one region, a rhombus, where tunneling involving real wave vectors is allowed. If these heights are different, then we have two such regions, one for each barrier. This is more clearly illustrated 


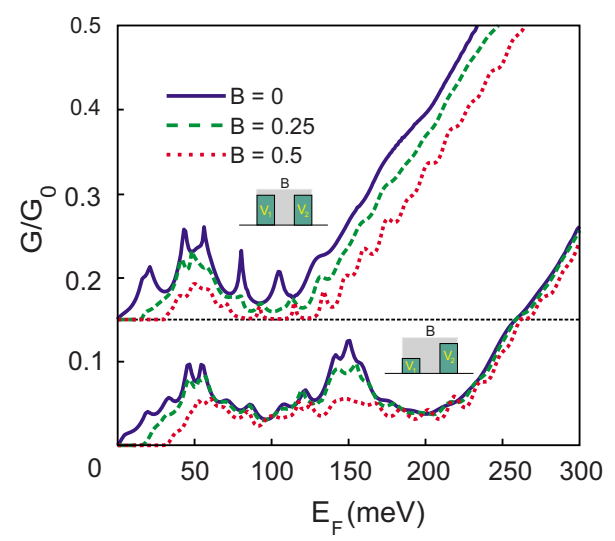

FIG. 16. (Color online) Conductance of a double barrier for different magnetic fields vs energy with $W=50 \mathrm{~nm}, W_{s}=50 \mathrm{~nm}$, and $V=100 \mathrm{meV}$.

in Figs. 17(a)-17(c), where we show, respectively, the conductance of two, three, and four asymmetric barriers shown at the bottom right of each panel. The other insets show pictorially these different transmission regions that are similar to that in Fig. 3. The vertical arrows pointing down in Fig. 17 and the corresponding parallel ones in the insets point to the same energies involved. Results to the right of the vertical arrows in the figure correspond to energies above the parallel arrows in the insets.

For completeness in Fig. 18 we show the conductance of a double, symmetric well (upper panel) and of an asymmetric one (lower panel) for positive energies $\left(E_{F}>0\right)$ with $W$ $=50 \mathrm{~nm}$, separation $W_{s}=50 \mathrm{~nm}, V_{1}=V_{2}=-100 \mathrm{meV}$. For clarity the results for the symmetric case are shifted up by 0.3 . Had we plotted the results for $E_{F}<0$, the picture would be the same as Fig. 16. However, as expected for $E_{F}>0$, there is not much difference between the results of the two panels.

One may wonder how the results given above change if we do not have abrupt interfaces of the rectangular barriers but instead consider, e.g., barriers or wells with smooth interfaces. We consider two such barriers, together with a square one, shown in the insets of Fig. 19. The second one is

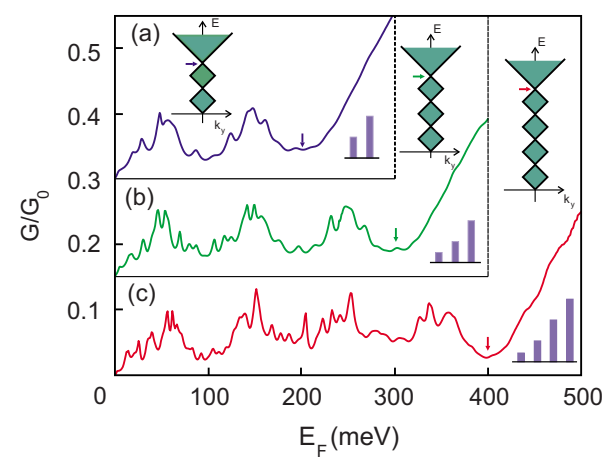

FIG. 17. (Color online) Conductance of three asymmetric structures as shown in the inset with $W=50 \mathrm{~nm}$ and separation $W_{s}=50 \mathrm{~nm}$. (a) $V_{2}=2 V_{1}=200 \mathrm{meV}$, (b) $V_{3}=3 V_{1}, V_{2}=2 V_{1}$, and $V_{1}=100 \mathrm{meV}$, and (c) $V_{4}=4 V_{1}, \quad V_{3}=3 V_{1}, \quad V_{2}=2 V_{1}$, and $V_{1}=100 \mathrm{meV}$.

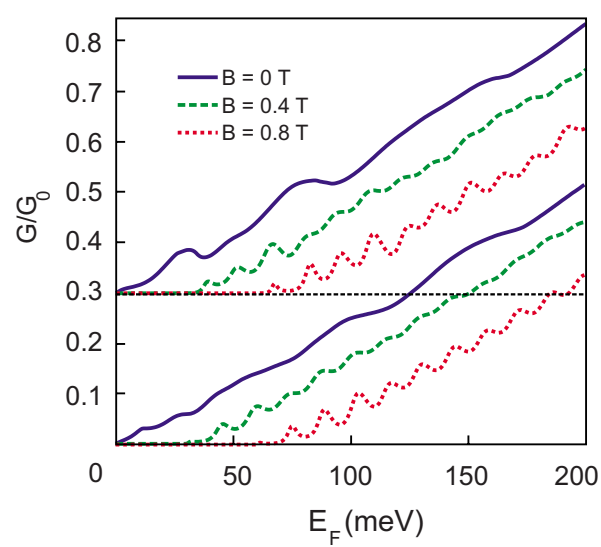

FIG. 18. (Color online) Conductance of a double well for different magnetic fields with well width $W=50 \mathrm{~nm}$, separation $W_{s}=50 \mathrm{~nm}$, and $V_{1}=V_{2}=-100 \mathrm{meV}$.

described by the semielliptic profile

$$
V(x)=\frac{2 V}{W}\left[W^{2} / 4-(x-W / 2)^{2}\right]^{1 / 2}
$$

and the third by the Gaussian profile

$$
V(x)=V \exp \left[-\left(2 \pi / W^{2}\right)(x-W / 2)^{2}\right],
$$

where $W$ is chosen such that the integral $\int V(x) d x$ has the same value for all three potential barriers.

To evaluate the transmission for such shapes we subdivide their spatial extent or width in several square barriers of different height and for each of them we use the results of Sec. III. Figure 19 contrasts the results of these shapes with those for a square barrier of height $V$ for zero field in the lower panel and $B=5 \mathrm{~T}$ in the upper one, which is shifted up by 0.15 for clarity. As can be seen, the results are qualitatively similar but the conductance for a square barrier is a bit larger when $E_{F}<V$ and has a less pronounced peak structure. The resonant structure on the other hand is quantitatively different for the three cases as expected but qualitatively there are clear similarities.

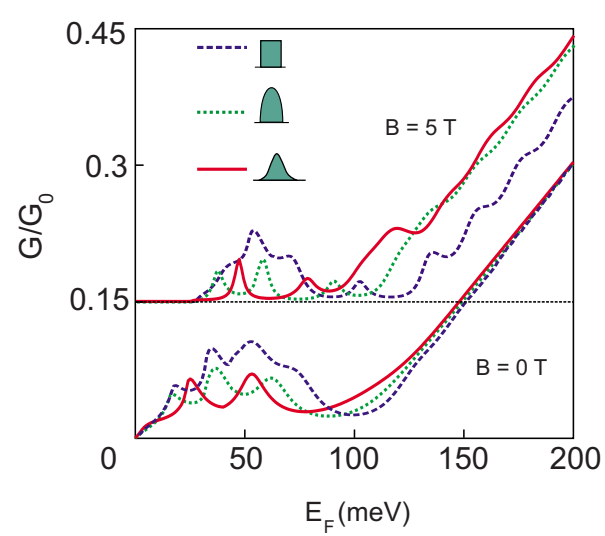

FIG. 19. (Color online) Conductance of the barriers shown in the inset for two different magnetic fields with $W=100 \mathrm{~nm}$ and $V=100 \mathrm{meV}$. 


\section{CONCLUDING REMARKS}

We evaluated the transmission and conductance through single and double potential barriers and wells in the absence or presence of a magnetic field. We also considered different shapes of barriers as well as a few barriers with different potential heights. We placed the emphasis on the influence of these factors on the Fabry-Pérot resonances, that is, we studied them as Fabry-Pérot interferometers.

We obtained the energies for which the tunneling probability is maximum for single and double barriers. The maxima in the transmission are connected with bound states, for energy smaller than $V / 2$, at $E= \pm k_{y}$.

When a magnetic field is applied the cyclotron motion leads to a decrease in the transmission and consequently in the conductance. The magnetic field dependence of the position of the resonances is very different from the nonrelativistic electron case: (1) their shift in energy is linear in magnetic field and (2) for $E<V$ they decrease with the field while for $E>V$ they increase.

For a single barrier we showed that the resonances in the transmission are reflected in those of the conductance.
Extra resonances are found for $E_{F}<V / 2$ which result from the connection between the maxima in the transmission and the bound states. For the special value $E_{F}=V$ we found that the conductance remains the same upon increasing the magnetic field and is given approximately by $G=\left(4 e^{2} / v_{F} h^{2}\right)(L / W) \tanh (V W)$.

We showed that using asymmetric barriers, with unequal heights, we can create several different transmission or conductance regions in energy space involving only real wave vectors whereas for symmetric barriers with equal heights, there is only one such region. We also evaluated the conductance of single barriers with smooth interfaces. We found that the oscillatory structure of the conductance becomes somewhat more pronounced but remains qualitatively the same as that of square barriers.

\section{ACKNOWLEDGMENTS}

This work was supported by the Flemish Science Foundation (FWO-V1), the Belgian Science Policy (IAP) and the Canadian NSERC under Grant No. OGP0121756. *mrmphys@gmail.com

takis@alcor.concordia.ca

†francois.peeters@ua.ac.be

${ }^{1}$ K. S. Novoselov, A. K. Geim, S. V. Morozov, D. Jiang, M. I. Katsnelson, I. V. Grigorieva, S. V. Dubonos, and A. A. Firsov, Nature (London) 438, 197 (2005).

${ }^{2}$ Y. Zhang, Y. W. Tan, H. L. Stormer, and P. Kim, Nature (London) 438, 201 (2005).

${ }^{3}$ M. I. Katsnelson, K. S. Novoselov, and A. K. Geim, Nat. Phys. 2, 620 (2006).

${ }^{4}$ V. V. Cheianov, V. Faĺko, and B. L. Altshuler, Science 315, 1252 (2007).

${ }^{5}$ O. Klein, Z. Phys. 53, 157 (1929).

${ }^{6}$ J. M. Pereira, Jr., V. Mlinar, F. M. Peeters, and P. Vasilopoulos, Phys. Rev. B 74, 045424 (2006).

${ }^{7}$ X. Chen and J.-W. Tao, Appl. Phys. Lett. 94, 262102 (2009).

${ }^{8}$ J. H. Bardarson, M. Titov, and P. W. Brouwer, Phys. Rev. Lett. 102, 226803 (2009).

${ }^{9}$ F. M. Peeters and A. Matulis, Phys. Rev. B 48, 15166 (1993); J. Reijniers, F. M. Peeters, and A. Matulis, ibid. 59, 2817 (1999).

${ }^{10}$ A. De Martino, L. Dell' Anna, and R. Egger, Phys. Rev. Lett. 98, 066802 (2007).

${ }^{11}$ M. Ramezani Masir, P. Vasilopoulos, and F. M. Peeters, Phys. Rev. B 79, 035409 (2009).

${ }^{12}$ M. Ramezani Masir, P. Vasilopoulos, and F. M. Peeters, Appl. Phys. Lett. 93, 242103 (2008).

${ }^{13}$ S. Park and H. S. Sim, Phys. Rev. B 77, 075433 (2008).

${ }^{14}$ L. Oroszlany, P. Rakyta, A. Kormanyos, C. J. Lambert, and J. Cserti, Phys. Rev. B 77, 081403(R) (2008).

${ }^{15} \mathrm{H}$. Xu, T. Heinzel, M. Evaldsson, and I. V. Zozoulenko, Phys. Rev. B 77, 245401 (2008).

${ }^{16}$ M. Ramezani Masir, P. Vasilopoulos, A. Matulis, and F. M. Peeters, Phys. Rev. B 77, 235443 (2008).

${ }^{17}$ S. Ghosh and M. Sharma, J. Phys.: Condens. Matter 21, 292204
(2009).

${ }^{18}$ A. H. Castro Neto, F. Guinea, N. M. R. Peres, K. S. Novoselov, and A. K. Geim, Rev. Mod. Phys. 81, 109 (2009); D. S. L. Abergel, V. Apalkov, J. Berashevich, K. Ziegler, and T. Chakraborty, Adv. Phys. 59, 261 (2010).

${ }^{19}$ J. Milton Pereira, Jr., P. Vasilopoulos, and F. M. Peeters, Appl. Phys. Lett. 90, 132122 (2007).

${ }^{20}$ S. Cho and M. Fuhrer, arXiv:0901.4157 (unpublished).

${ }^{21}$ M. Dragoman, D. Dragoman, G. Deligiorgis, G. Konstantinidis, D. Neculoiu, A. Cismaru, and R. Plana, J. Appl. Phys. 106, 044312 (2009).

${ }^{22}$ A. V. Shytov, M. S. Rudner, and L. S. Levitov, Phys. Rev. Lett. 101, 156804 (2008).

${ }^{23}$ A. F. Young and P. Kim, Nat. Phys. 5, 222 (2009).

${ }^{24}$ J. Velasco, Jr., G. Liu, W. Bao, and C. N. Lau, New J. Phys. 11, 095008 (2009).

${ }^{25}$ R. V. Gorbachev, A. S. Mayorov, A. K. Savchenko, D. W. Horsell, and F. Guinea, Nano Lett. 8, 1995 (2008).

${ }^{26}$ V. V. Cheianov and V. I. Faĺko, Phys. Rev. B 74, 041403(R) (2006).

${ }^{27}$ B. Özyilmaz, P. Jarillo-Herrero, D. Efetov, D. A. Abanin, L. S. Levitov, and P. Kim, Phys. Rev. Lett. 99, 166804 (2007).

${ }^{28}$ B. Huard, J. A. Sulpizio, N. Stander, K. Todd, B. Yang, and D. Goldhaber-Gordon, Phys. Rev. Lett. 98, 236803 (2007).

${ }^{29}$ J. R. Williams, L. DiCarlo, and C. M. Marcus, Science 317, 638 (2007).

${ }^{30}$ J. B. Oostinga, H. B. Heersche, X. L. Liu, A. F. Morpurgo, and L. M. K. Vandersypen, Nature Mater. 7, 151 (2008).

${ }^{31}$ G. Liu, J. Velasco, W. Z. Bao, and C. N. Lau, Appl. Phys. Lett. 92, 203103 (2008).

${ }^{32}$ V. A. Yampol'skii, S. Savel'ev, and F. Nori, New J. Phys. 10, 053024 (2008)

${ }^{33}$ I. S. Gradshteyn and I. M. Ryzhik, Handbook of Mathematical Functions (Academic Press, New York, 2000), p. 1021. 
${ }^{34}$ P. G. Silvestrov and K. B. Efetov, Phys. Rev. Lett. 98, 016802 (2007).

${ }^{35}$ N. Gu, M. Rudner, A. Young, P. Kim, and L. Levitov, arXiv:1003.2399 (unpublished); A. Kormányos, P. Rakyta, L. Oroszlány, and J. Cserti, Phys. Rev. B 78, 045430 (2008); P.
Carmier and D. Ullmo, ibid. 77, 245413 (2008).

${ }^{36}$ J. M. I. Katsnelson, Eur. Phys. J. B 51, 157 (2006).

${ }^{37}$ J. Tworzydło, B. Trauzettel, M. Titov, A. Rycerz, and C. W. J. Beenakker, Phys. Rev. Lett. 96, 246802 (2006). 